Trauma Berufskrankh 2017· [Suppl 1]: 19:S67-S69 DOI 10.1007/s10039-017-0230-5

Online publiziert: 31. Januar 2017

๑) Springer Medizin Verlag Berlin 2017

CrossMark

\section{B. Friemert}

Klinik für Unfallchirurgie und Orthopädie, Rekonstruktive und Septische Chirurgie, Sporttraumatologie, Bundeswehrkrankenhaus Ulm, Akademisches Krankenhaus der Universität Ulm, Ulm, Deutschland
Weltweit gehört das Trauma zu den 10 häufigsten Todesursachen. Aktuell gibt es weltweit ca. 5,8 Mio. Verletzte jährlich. Die Hochrechnungen zeigen, dass es im Jahr 2020 ca. 8 Mio. Patienten sein werden. Allein in der EU geht man von 1 Mio. Toten aus. Die 3 Hauptursachen sind Unfälle jeglicher Art, der Suizid und auch Tötungsdelikte.

Bei Betrachtung der Todesursachen stellt man fest, dass die häufigste Todesursache bei Unfällen und Suiziden die Blutung ist. Etwa 30-40 \% der Verstorbenen verbluten in der Akutphase. Somit stellen die Blutung und damit der hämorrhagische Schock einen wesentlichen Grund des traumabedingten Versterbens dar.

Bei den Überlebenden spielt neben dem hämorrhagischen Schock die Entwicklung einer Koagulopathie eine zentrale Rolle für das Versterben. So zeigen ca. $25 \%$ der schwer verletzten Patienten bei der Ankunft im Krankenhaus schon eine Koagulopathie; 84 \% von diesen Patienten hatten einen Injury Severity Score (ISS) von $>16$ und sind damit schwer traumatisiert.

Die Folge dieser Koagulopathie ist eine deutliche erhöhte Rate an Multiorganversagen, das eine wesentliche sekundäre Todesursache im Verlauf der Behandlung ist. Somit spielt die Blutungskontrolle bei der Frage des akuten Überlebens, aber auch bei der Frage der Entwickelung eines Multiorganversagens in der Frühphase der Behandlung eines traumatisierten Patienten eine überragende Rolle.

„Stop the bleeding“ ist der Schlüsselaufgabe für das Senken der traumatisch bedingten hohen Letalität.

Die wesentliche Bedeutung der Hämorrhagie wird auch im Rahmen der ATLS-Ausbildung deutlich, wo an drit- ter Stelle des Behandlungsalgorithmus die Frage der Hämorrhagie und damit die Entwicklung des hämorrhagischen Schocks und nachfolgend der Koagulopathie eine wesentliche Rolle spielt. Der Frage stabiler vs. instabiler Patient kommt von Anfang an bei diesen Patienten im Rahmen des Advanced Trauma Life Support(ATLS)-Konzeptes eine überragende Bedeutung zu.

Aus dieser Frage ergibt sich dann im Schockraum die Entscheidung entweder zur lebensrettenden Sofortoperation im Sinne einer Damage Control Surgery oder zu einer weitergehenden Diagnostik bis hin zum „secondary survey“ und ggf. zunächst zur Stabilisierung des Patienten auf der Intensivstation oder zu sekundär frühen operativen Verfahren, wie z.B. die knöcherne Stabilisierung mittels Fixateur extern. Somit spielt die Frage der Blutungskontrolle in den ersten $30 \mathrm{~min}$ nach Aufnahme des Patienten in den Schockraum die entscheidende Rolle für die weitere Therapie des Patienten.

In der 4. Edition liegen nun die europäischen Guidelines zum Management schwerer Blutungen und der Koagulopathie nach Trauma vor. Diese sind veröffentlicht von Rossaint et al. [1]. Für jeden Traumatologen lohnt es sich, diese Guidelines zu lesen, da hier wesentliche pathophysiologische wie auch klinische Informationen zum Thema „Stop the bleeding" zu finden sind.

Im Zentrum der Betrachtung massiver Blutungen steht die tödliche Trias, die sich durch einen erheblichen Blutverlust mit folgender Hyperthermie, Azidose und Koagulopathie ergibt. Um diese tödliche Trias zu beenden und zu unterbrechen, ist eine schnellstmögliche und effektive Blutungskontrolle zwingend er- forderlich. Die Gabe von Blutprodukten und andere stabilisierende Maßnahmen sind in der Regel nicht in der Lage, bei schweren Hämorrhagien diesen Kreislauf dauerhaft zu durchbrechen.

Als zentrales Problem entwickelt sich die Koagulopathie, deren Ursache zum einen in erheblichen Gewebetraumata, vor allen Dingen der Weichteile, zu suchen ist, da alleine hierdurch eine erhebliche Gerinnungsaktivierung erfolgen kann.

Weiterhin entstehen durch die Blutung eine Hypoperfusion, letztlich auch eine Hypothermie und als Folge dieser Mechanismen eine Azidose. Eine Inflammation, die bei schweren Traumata regelhaft dazukommt, verstärkt die Koagulopathie noch, genauso wie die im Rahmen der Therapie durchgeführte Hämodilution.

\section{Azidose}

Die Azidose wird repräsentiert durch den Base-Excess und das Laktat. Ein Base-Excess von $>-6 \mathrm{mmol} / \mathrm{l}$ verursacht in $2 \%$ der Fälle eine Gerinnungsstörung, ein Base-Excess von <-6 mmol/l ist vergesellschaftet mit einer 20\%igen Gerinnungsstörung. Das Gleiche gilt für Laktatwerte von $>$ bzw. $<2,2 \mathrm{mmol} / \mathrm{l}$.

Die Azidose wird im Wesentlichen durch die schlechte Gewebeperfusion aufgrund der Hämorrhagie hervorgerufen, wodurch ein anaerober Stoffwechsel entsteht. Dieser reduziert die kardiale Leistung und vermindert auch die Katecholaminwirkung, sodass hierdurch der schon durch die Hämorrhagie instabile Kreislauf weiter geschwächt wird. Bei einer Veränderung des $\mathrm{pH}$ von 7,4 auf 7,0 wird die Wirksamkeit der Ge- 
rinnungskaskade um 50-70\% reduziert, sodass Gerinnungsfaktoren, z. B. der Faktor VIIa, nicht mehr wirken können, was dann wiederum die Hämorrhagie verstärkt.

\section{Hypothermie}

Bei vorliegender Hypothermie ist zur Wärmegewinnung erhebliche Mengen Adenosintirphospat (ATP) erforderlich, das im Rahmen der Azidose in deutlich reduzierter Menge vorliegt. Die Hypothermie reduziert ebenfalls die Effektivität der Gerinnungskaskade und die Plättchenfunktion. Dadurch verstärkt sich wiederum die Blutung.

Ein Hauptgrund für die Hypothermie ist oft eine zu niedrige Raum- bzw. Umgebungstemperatur, aber auch eine Flüssigkeitszufuhr mit kalten Infusionen. Durch den Verbrauch von Gerinnungsfaktoren, wie oben beschrieben, und die Hämodilution exazerbiert dann die Koagulopathie. Ein hoher Trauma-Load verbraucht erhebliche Mengen an Gerinnungsfaktoren, genauso wie ein schweres Gewebetrauma.

\section{Weitere die Koagulopathie beeinflussende Faktoren}

Die Koagulopathie wird zusätzlich noch durch folgende Faktoren beeinflusst:

Schädel-Hirn-Trauma (SHT), Alter, genetische Faktoren, Medikamente, Vorerkrankungen, gerinnungshemmende Medikamente.

\section{Entwicklung der Koagulopathie}

Betrachtet man nun die Entstehung der Koagulopathie nach Trauma, so wird sehr schnell deutlich, dass unmittelbar nach der Gewebeschädigung Zytokine und Hormone ausgeschüttet werden, die unmittelbar mit der Traumatisierung zusammenhängen. Diese Faktoren beeinflussen ganz erheblich die Entstehung der Koagulopathie. Allerdings ist diese Seite der Entwicklung der Koagulopathie nicht beeinflussbar, da sie durch das Trauma selbst bedingt ist.

Der zweite Hauptaspekt zur Entwicklung einer Koagulopathie ist die Hämorrhagie, wie oben dargestellt. Genau dieser

Trauma Berufskrankh 2017 · [Suppl 1]: 19:S67-S69 DOI 10.1007/s10039-017-0230-5

(c) Springer Medizin Verlag Berlin 2017

B. Friemert

„Stop the bleeding" beim Traumapatienten

\section{Zusammenfassung}

Weltweit gehört das Trauma zu den 10 häufigsten Todesursachen. Allein in der EU geht man von 1 Mio. Toten aus. Bei Betrachtung der Todesursachen stellt man fest, dass die häufigste Todesursache bei diesen Unfällen die Blutung ist. Etwa 30-40 \% der Verstorbenen verbluten in der Akutphase. Somit stellen die Blutung und damit der hämorrhagische Schock einen wesentlichen Grund des traumabedingten Versterbens dar. Bei den Überlebenden spielt neben dem hämorrhagischen Schock die Entwicklung einer Koagulopathie eine zentrale Rolle für das Versterben. So zeigen ca. $25 \%$ der schwer verletzten Patienten bei der Ankunft im
Krankenhaus schon eine Koagulopathie. Die Folge dieser Koagulopathie ist eine deutlich erhöhte Rate an Multiorganversagen, das eine wesentliche sekundäre Todesursache im Verlauf der Behandlung ist. Somit spielt die Blutungskontrolle bei der Frage des akuten Überlebens, aber auch bei der Frage der Entwickelung eines Multiorganversagens in der Frühphase der Behandlung eines traumatisierten Patienten eine überragende Rolle.

\section{Schlüsselwörter}

Koagulopathie · Todesursache · Unfall · Hämorrhagischer Schock · Multiorganversagen

\section{Stop the bleeding in trauma patients}

\section{Abstract}

Trauma is one of the ten most common causes of death worldwide and in the European Union (EU) alone, it is assumed that there are one million deaths. When the causes of death are considered it is found that the most frequent cause of death in these accidents is bleeding. Of the deceased $30-40 \%$ bleed to death in the acute phase; therefore, hemorrhage and thus hemorrhagic shock, constitute a fundamental cause of traumatic death. In addition to hemorrhagic shock, the development of a coagulopathy plays a central role for survivors. Approximately $25 \%$ of severely injured patients show a coagulopathy on arrival in hospital. The consequence of this coagulopathy is a markedly increased rate of multiple organ failure, which is a major secondary cause of death in the course of the treatment. Thus, control of bleeding plays an important role not only in the question of acute survival but also in the development of multiple organ failure in the early phase of treatment of trauma patients.

Keywords

Coagulopathy - Cause of death - Accident . Hemorrhagic shock · Multiple organ failure ist durch chirurgisches Handeln beeinflussbar und muss schnellstmöglich beseitigt werden, um eine Koagulopathie entweder zu verhindern oder die tödliche Trias, die die Koagulopathie hervorruft, $\mathrm{zu}$ unterbrechen.

\section{Blutungskontrolle als wichtigste Maßnahme}

Die Bedeutung einer vorliegenden Koagulopathie in Bezug auf die Letalität zeigt sich durch verschiedene Studien, die im Deutschen Ärzteblattheft 49 am 09.12.2011 [2] veröffentlicht wurden. Es zeigt sich, dass mit Vorliegen einer Koagulopathie die Letalität um den Faktor 4,6 erhöht ist. Somit ist klar, dass die
Hämorrhagie der Faktor ist, der durch chirurgisches Handeln beeinflussbar ist, dadurch die Koagulopathie beeinflusst werden kann und somit auch die Letalität gesenkt wird.

Dies macht deutlich, dass „Stop the bleeding" im Rahmen der Traumabehandlung in der Frühphase im Schockraum die zentrale Rolle spielt. Die Beherrschung der Hämorrhagie und die damit verbundene Blutungskontrolle sind notwendig, um die Gewebeperfusion zu halten bzw. schnellstmöglich wieder herzustellen. Die Blutstillung kann entweder chirurgisch oder in manchen Regionen heute besser interventionell erfolgen. Alle Bemühungen am blutenden Patienten müssen darauf ausgerichtet sein, 
zunächst eine Blutungskontrolle $\mathrm{zu}$ erreichen. „Stop the bleeding“ ist Teil des Konzeptes der Damage Control Surgery.

Therapeutisch sind in der Präklinik folgende Optionen verfügbar: Abdrücken großer Arterien, Druckverband, Tourniquet, Hämostyptika sowie eine Ligatur bzw. im Ausnahmefall auch Klemmen.

Aktuell spielen sicherlich der Druckverband, das Tourniquet und die Hämostyptika eine ganz zentrale Rolle bei der präklinischen Blutungskontrolle. Wichtig ist an dieser Stelle zu sagen, dass der Druckverband wie auch das Tourniquet grundsätzlich so stark angezogen werden müssen, bis distal eine entsprechende Blutungskontrolle vorliegt. Ein zu gering angezogenes Tourniquet führt eher zu einer venösen Stauung und damit zu einer Vergrößerung der Blutungsproblematik.

Die Blutungskontrolle im Gesichtsund Halsbereich kann über Tamponaden oder aufblasbare Ballonkatheter, wie z. B. ein Urinkatheter, erfolgen. Die thorakale Blutungskontrolle erfolgt entweder über eine Thoraxdrainage (in $90 \%$ der Fälle ausreichend) oder eine Thorakotomie mit z. B. Ligatur einer Interkostalarterie oder einer atypischen Lungenresektion.

Im Abdomen erfolgt die Blutungskontrolle entweder durch Packing parenchymatöser Organe oder durch die Ligatur bzw. Rekonstruktion entsprechender anatomisch verletzter Strukturen bis hin zur Entfernung von Organen, wie z. B. der Milzexstirpation.

Im Bereich des Beckens spielen vor allen Dingen bei den C-Verletzungen Blutungen im kleinen Becken im Bereich des präsakralen Plexus eine überragende Rolle. Hier ist es zwingend erforderlich, eine schnellstmögliche anatomische Stabilisierung des Beckens (Schwerpunkt dorsaler Beckenring) zu erreichen, entweder mit einem Fixateur extern, einem Pelvic Sheeting oder der Beckenzwinge. Zusätzlich kann dann suprapubisch noch ein Packing des kleinen Beckens durchgeführt werden.

Im Hinblick auf die Blutung von Extremitäten sollte präsent sein, dass auch geschlossene Frakturen erheblich bluten können. So können bei einer Femurfraktur in den Oberschenkel ca. 21 Blut versacken. Somit kann auch ohne Blutaustritt nach außen durch multiple Frakturen ein hämorrhagischer Schock entstehen. Die beste Therapie hierzu ist die schnellstmögliche temporäre Stabilisierung der Fraktur mittels Fixateur extern und einer möglichst genauen Reposition, allerdings ohne sich mit Details zu lange aufzuhalten, denn das primäre Ziel ist es, den Patienten möglichst schnell auf die Intensivstation zur Stabilisierung zu verbringen.

Ein aktuelles neues Verfahren, um eine proximale Blutungskontrolle bei Patienten in extremis zu erreichen, ist das Reboa-Verfahren. Hierbei wird über die Leiste ein Ballonkatheter in die Aorta descendens eingebracht, entweder thorakal oder abdominal platziert, je nach Blutungsquelle. Dieser Ballon wird dann befüllt, sodass die Aorta okkludiert wird und somit eine distale Blutungskontrolle erreicht werden kann.

\section{Zusammenfassung und Schlussfolgerung}

Im Rahmen des Traumas spielt die Hämorrhagie bei der Frage der Inzidenz der Letalität eine überragende Rolle. Die Hämorrhagie ist ein wesentlicher Faktor zur Entwicklung einer tödlichen Trias, die sich aus Azidose, Hypothermie und Koagulopathie zusammensetzt.

In der ersten Phase der Behandlung eines Patienten im hämorrhagischen Schock ist es daher notwendig, so schnell wie möglich eine Blutungskontrolle $\mathrm{zu}$ erreichen. Hierzu sind in der Regel keine aufwendigen und komplexen Verfahren erforderlich, sodass gut ausgebildeten Chirurgen die erste operative Blutstillung gelingen sollte. Jede Blutstillung ist besser als keine Blutstillung, und Blutstillung ist letztlich eine ureigene chirurgische Tätigkeit.

\section{Fazit für die Praxis}
Aufgrund der zentralen Bedeutung des hämorrhagischen Schocks inklusive der posttraumatischen Koagulopathie beim Traumapatienten ist die Blutungskon- trolle die zentrale und lebensrettende therapeutische Maßnahme. Dies gilt für die Präklinik wie für die klinische Be-

handlung. "Stop the bleeding" rettet Leben!

\section{Korrespondenzadresse}

Prof. Dr. med. B. Friemert

Klinik für Unfallchirurgie und Orthopädie, Rekonstruktive und Septische Chirurgie, Sporttraumatologie, Bundeswehrkrankenhaus Ulm, Akademisches Krankenhaus der Universität Ulm

Oberer Eselsberg 40, 89081 Ulm, Deutschland benediktfriemert@t-online.de

\section{Einhaltung ethischer Richtlinien}

Interessenkonflikt. B. Friemert gibt an, dass kein Interessenkonflikt besteht.

Dieser Beitrag beinhaltet keine vom Autor durchgeführten Studien an Menschen oder Tieren.

The supplement containing this article is not sponsored by industry.

\section{Literatur}

1. Rossaint R, Bouillon B, Cerny V, Coats TJ, Duranteau J, Fernández-Mondéjar E (2016) The European guideline on management of major bleeding and coagulopathy following trauma: fourth edition. $\mathrm{Cr}$ cal Care 20:100. doi:10.1186/s13054-016-1265-x

2. Maegele M, Paffrath T, Bouillon B (2011) Akute trauma-assoziierte Gerinnungsstörung beim Schwerverletzten. Dt Ärztebl 108(49):827-835 